\title{
Abnormal WBC Scattergrams by Sysmex XN550, A Supplementary Diagnostic Tool for Malaria to the Conventional Methods
}

\author{
Rajeshwar Suresh Bute ${ }^{1}$, BhavanaMadhukar Bharambe ${ }^{1}$, RavikiranPawar ${ }^{2}$ and Asawari Bharat Jadhav ${ }^{1}$ \\ ${ }^{1}$ Grant Government Medical College \& Sir J.J. Group of Hospitals, Mumbai, India. \\ ${ }^{2}$ SRL Diagnostics,Mumbai. India
}

\begin{abstract}
Background: In India, malaria has a major impact on healthsystem.It is usually diagnosed based on symptomatology, parasite detection in the peripheral smear (PS) or rapid diagnostic tests (RDT) such as malaria antigen test(MAT). Detection of malaria by MAT is considered as the gold standard. A rapid, cost effective screening of malaria can be done with the automated analyzers. The present study was undertaken to assess the efficacy of WBC scattergram generated by SysmexXN550 hematologyanalyzer to diagnose malaria.

Methods: A prospective study was conducted over a period of 4 months from August to November2019, after obtaining institutional ethical clearance.All cases diagnosed as Plasmodium vivax/Plasmodium falciparum infections on malaria antigen test (MAT) were included. Their hemogram and WBC scattergrams obtained from Sysmex XN 550 were studied. Thick \& thin Smears were made and stained with Leishman's stain for microscopy.

Results: A total of 101 cases were diagnosed as malaria positive by MAT and thick smear.Ninety-sevenwere positive by Leishman's stain. Abnormal scattergramswere 81 out of 101 malaria positive cases. The commonest pattern was double neutrophil zone( $\mathrm{n}=22$ ) followed by double neutrophil with less space between neutrophil and eosinophil ( $\mathrm{n}=17)$. An abnormal event on $\mathrm{X}$ axis was observed in 16 patients. Gray zone and double eosinophil areas were observed in 11 and 4 cases respectively. The sensitivity of the analyzer was found to be $80.19 \%$.
\end{abstract}

Conclusion: Scattergram of automated haematology analyser(Sysmex XN550) has good sensitivity, which can be increased to a better level if combined with thrombocytopenia and symptomatology of the patients.

Keywords: Scattergram, Malaria parasite, Sysmex, Thrombocytopenia, Hematology Analyzer

\section{Introduction}

Malaria is one of the most common parasitic diseases in the world. It is caused by parasites of the genus Plasmodium, which infects human being through the bite of mosquitoes of the genus Anopheles. Clinical diagnosis of malarial infection is based on patient's signs and symptoms such as fever with chills and as these are very nonspecific correct diagnosis becomes difficult..$^{[1]}$ The microscopic detection of malaria parasite is the standard method of diagnosis till now. Though this is a cheaper method, there is a high degree of subjectivity while reporting of the smears. Accurate interpretation of the peripheral smear (PS) needs considerable expertise. So, this method becomes less reliable in cases with low parasitic index. Hence, laboratory misdiagnosis is may occur. Hematology analyzers are now being routinely used in many laboratories. The automation in hematology helps in analyzing various parameters. The normal scattergram (Fig:1) in the DIFF plot consists of five components: lymphocytes (pink), monocytes (green), neutrophil and basophils (blue), eosinophils (red) and a space between the neutrophil and eosinophil populations. ${ }^{[2]}$

Nowadays many automated analysers are available which can detect malarial parasite if careful observation of scattergram is done. It is found that hemazoin pigment produced by malaria also scatter the laser light, producing abnormal scattergrams in CBC analysis.

Different abnormal patterns found on WBC-DIFF (differential) scattergrams (Fig:2) in malaria infection are mixing of neutrophils \& eosinophils clusters, double neutrophils or double eosinophils clusters, graying of neutrophils \& eosinophils clusters, reduced space between eosinophils \& neutrophils clusters, large eosinophils clusters and prominent blue/purple events above $\mathrm{X}$ axis. ${ }^{[3]}$

Hemozoin pigments produced by malaria parasite have property to depolarize the laser light. So, the infected RBCs having different morphologic forms eg.trophozoites(also known as ring forms), schizoints, gametocyteand the phagocytic cells(monocyte, macrophage, and neutrophil containing the parasite) produce bizzare and unusual scattergrams during routine $\mathrm{CBC}$ analysis. ${ }^{[4]}$

The present study was conducted to check the efficacy of WBC scattergrams by Sysmex XN 550 to diagnose malaria so that early diagnosis and treatment prevents the subsequent complications. 


\section{Materials and Methods:}

This is a prospective study and carried over an interval of 4 months from August 2019 to November 2019 after approval by an ethical committee. Samples of the patients of all age groups who were sent for investigations by clinicians to hematology section of our tertiary care hospital were collected. Only those which were positive for Plasmodium Vivax and Plasmodium Falciparum by MAT test were included in our study. Blood samples were collected in an EDTA bulb and processed on the Sysmex XN 550 differential cell counter in hematology section of the department of Pathology. Complete blood counts including all parameters of $\mathrm{RBC}, \mathrm{WBC}$ and platelets were assessed. WBC scattergrams were studied in each case. Clinical history of all the patients was obtained. Smears of the fresh samples were made and stained with Leishman's stain for microscopy. Also, thick smears were prepared for all the cases. Sensitivity of scattergram, thin smear and thick smear was assessed and compared with that of MAT.

\section{Source of data}

The OPD and in patients of tertiary care hospital from August 2019 to November 2019

\section{Result}

Out of total 101 malaria cases 67 were males while 34 were females. The age range of the patients varied between 2 months and 81 years with maximum patients in the age group of 21 to 30 years. $P$. vivax cases were predominant accounting for 93 whereas $P$. falciparum were 5 and 3 cases of mixed infection of vivax and falciparum were found (Table 1). A total of 101 cases were diagnosed as malaria positive by MAT and thick smear. Ninety-seven amongst them were found positive for malaria by Leishman-stained thin film (Table 2). Abnormal scattergrams were shown by the analyzer in $81(80.19 \%)$ out of 101 malaria positive cases. The commonest pattern was double neutrophil zone $(n=22) 27.16 \%$ followed by double neutrophil with less space between neutrophil and eosinophil $(n=17)$ $20.98 \%$. An abnormal event on $\mathrm{X}$ axis was observed in $(n=16) 19.75 \%$ patients. Gray zone and double eosinophil areas were observed in $n=11(13.58 \%)$ and $n=4(4.93 \%)$ cases respectively (Table 3 ). The sensitivity of the analyzer was found to be $80.19 \%$.

\section{Hematological findings: (Table 1)}

Hemogram study revealed thrombocytopenia in $93.06 \%$ cases, anaemia and leucopenia in 58.41\% and16.83\% cases respectively. Pancytopenia was observed in $9.9 \%$ and leukocytosis in $22.77 \%$ case and pseudoeosinophilia in $19.80 \%$.

\section{Discussion}

Scattergram is a graph of the distribution of two variables in a sample population. One variable is plotted on the Y axis; the second is plotted on the $\mathrm{X}$ axis. A scattergram shows the degree or tendency with which the variables occur in association with each other. The fluorescence flow cytometry principle is used by the Sysmex analysers. In the fluorescence flow cytometry technology, cells are subjected to a laser beam which gets scattered in various directions. The forward scattered light measures the cell volume while the side scattered light measures cell granularity. ${ }^{[2]}$ Researchers have provided different patterns of scattergrams in patients with malaria which are as follows.

1. Abnormal events over $\mathrm{X}$ axis (marked by purple color)

2. Mixing of eosinophils and neutrophils scatter plots. which may be orange-red, sky blue or graying color.

3. Reduced space between neutrophil and eosinophil groups.

4. Appearance of multiple eosinophil or neutrophil groups.

5. Mixed patterns. ${ }^{[5,6,7]}$

In present study $n=81(80.19 \%)$ of the scattergrams were abnormal suggesting malarial infection whereas $n=20$ (19.80\%) was normal. Out of 101 positive cases all showed parasite on thick PS, while on thin smear $n=97(96.03 \%)$ was positive, those 4 cases which were missed on thin smear had low parasite index.

Out of 20 cases which were normal on scattergram, all were having symptoms of fever with chills and 16 of them had thrombocytopenia.

Sharma Sunita et al ${ }^{[8]}$ in their study had sensitivity of $83.58 \%$ and Mohapatra et al ${ }^{[9]}$ had $74.28 \%$, both of which were in concordance with our study. While B S Ramya et al ${ }^{[10]}$ obtained scattergram sensitivity of $90.5 \%$ to detect malaria. (Table 4)

While analyzing the WBC scattergrams, we found the commonest pattern was double neutrophil zone $27.16 \%$ followed by double neutrophil with less space between neutrophil and eosinophil $20.98 \%$ both of these patterns were showing late trophozoites and schizonts in peripheral smear. A study by Paividya et al. ${ }^{[11]}$ also reported similar microscopic findings with double neutrophil pattern. An abnormal event on $\mathrm{X}$ axis was next common about $19.75 \%$ patients. Gray zone and double eosinophil areas were observed in $n=11(13.58 \%)$ and $n=4(4.93 \%)$ cases respectively. It was also observed that scattergram of the patients with malaria returned to the normal after completion of the treatment (Fig:3). 
On the contrary in study by Sharma $\mathrm{S}$ et al ${ }^{[8]}$, most common abnormality was found to be graying of eosinophil and neutrophil populations $(41.10 \%)$ which was $13.58 \%$ in our study.

In course of our study, we also focused on PS findings, prevalence of anaemia, thrombocytopenia, pseudoeosinophilia and clinical features. Different stages of parasites were noticed such as trophozoites (Fig:4a), schizonts (Fig:4b) and gametocytes (Fig:4c).

Malaria can be predicted in patients who present with a combination of anemia and thrombocytopenia. ${ }^{[12]}$ Gupta $P$ et al ${ }^{[13]}$ found that thrombocytopenia was significantly seen in $89.3 \%$ of malaria cases. Thrombocytopenia was present in $97.7 \%$ of malaria positive cases, according to study by Hassan Mubin et al [14] Similar findings were observed by Abro et al ${ }^{[15]}$ and Chandra et al. ${ }^{[16]}$ In present study, we also had similar findings with $93.06 \%$ cases of thrombocytopenia. Evolution of anemia in malaria is complex and depends on many factors. It occurs due to hemolysis of infected RBCs, depressed as well as ineffective erythropoiesis with dyserythropoiesis and anemia of chronic disease. ${ }^{[17,18]}$ In our study $58.41 \%$ patients were anemic.

Sometimes, a spurious increase in the mixed cell population (both monocytes and eosinophils) can be a clue to the presence of malaria parasites in the red blood cells. [19] This is thought to occur because the parasite infected RBCs cannot be lysed by the diluent solution and will enter the WBC counting chamber. In a study by Adlekha et al ${ }^{[20]}$ using Sysmex XS-800i analyzer, a spurious increase in the mixed cell population was found to be moderately sensitive and highly specific in diagnosing malaria. In our study pseudoeosinophilia was found to be $19.80 \%$.

Table 1: Hemogram findings in malaria cases.

\begin{tabular}{|l|l|l|}
\hline Hemogram abnormality & $\begin{array}{l}\text { Number of malaria cases } \\
\text { N-101(vivax-93, falci-5, vivax+falci-3) } \\
(\text { Male-67, Female-34) }\end{array}$ & Percentage\% \\
\hline Leucopenia $(<4000 / \mu \mathrm{l})$ & 17 & 16.83 \\
\hline Anaemia only $(\mathrm{M}<13 \mathrm{gm} / \mathrm{dl}, \mathrm{F}<12 \mathrm{gm} / \mathrm{dl})$ & 59 & 58.41 \\
\hline Thrombocytopenia only $(<1,50,000 / \mu \mathrm{l})$ & 94 & 93.06 \\
\hline Pancytopenia & 10 & 9.9 \\
\hline Leukocytosis & 23 & 22.77 \\
\hline Pseudoeosinophilia & 20 & 19.80 \\
\hline
\end{tabular}

Table 2: Sensitivity in diagnosing malaria cases.

\begin{tabular}{|l|l|l|l|}
\hline \multirow{2}{*}{$\begin{array}{l}\text { Cases diagnosed on } \\
\text { scattergram }\end{array}$} & \multicolumn{2}{|l|}{ Cases diagnosed on peripheral smear } & \multicolumn{2}{l}{$\begin{array}{l}\text { Cases diagnosed by MAT } \\
\text { (malaria antigen test) }\end{array}$} \\
\cline { 2 - 4 } & On thin smear & On thick smear & $101(100 \%)$ \\
\hline $81(80.19 \%)$ & $97(96.03 \%)$ & $101(100 \%)$ & 100 \\
\hline
\end{tabular}

Table:3 Various abnormalities in WBC scattergram in malaria cases.

\begin{tabular}{|c|c|c|c|c|c|}
\hline Scattergram finding & P. vivax & P. falciparum & $\begin{array}{l}\text { PV + PF mixed } \\
\text { infection }\end{array}$ & $\begin{array}{l}\text { Total } \\
\text { N (\%) }\end{array}$ & $\begin{array}{l}\text { Predominant parasite } \\
\text { form }\end{array}$ \\
\hline Double neutrophil & 20 & 2 & 0 & $22(27.16 \%)$ & $\begin{array}{l}\text { Late trophozoite \& } \\
\text { schizont }\end{array}$ \\
\hline $\begin{array}{l}\text { Double neutrophil + Reduced } \\
\text { space }\end{array}$ & 15 & 1 & 1 & $17(20.98 \%)$ & $\begin{array}{l}\text { Late trophozoite \& } \\
\text { schizont }\end{array}$ \\
\hline Abnormal finding on $\mathrm{X}$ axis & 15 & 0 & 1 & $16(19.75 \%)$ & Early trophozoite \\
\hline Reduced space & 10 & 0 & 0 & $10(12.34 \%)$ & $\begin{array}{l}\text { Early trophozoite \& } \\
\text { schizont }\end{array}$ \\
\hline $\begin{array}{l}\text { Gray zone in neutrophil \& } \\
\text { Eosinophil }\end{array}$ & 11 & 0 & 0 & $11(13.58 \%)$ & Schizont\& gametocyte \\
\hline Double eosinophil & 3 & 1 & 0 & $4(4.93 \%)$ & Schizont \\
\hline Mixed pattern & 1 & 0 & 0 & $1(1.23 \%)$ & Trophozoite \& schizont \\
\hline Total abnormal cases & 75 & 4 & 2 & 81 & \\
\hline
\end{tabular}


Table 4: Comparative Scattergram studies in malaria cases.

\begin{tabular}{|c|l|l|}
\hline S. No. & Study & Sensitivity of Scattergram \\
\hline 1 & Sharma Sunita et al & $83.58 \%$ \\
\hline 2 & Mohapatra S et al & $74.28 \%$ \\
\hline 3 & Ramya et al & $90.5 \%$ \\
\hline 4 & Pai Vidya et al & $76.88 \%$ \\
\hline 5 & Present study & $80.19 \%$ \\
\hline
\end{tabular}

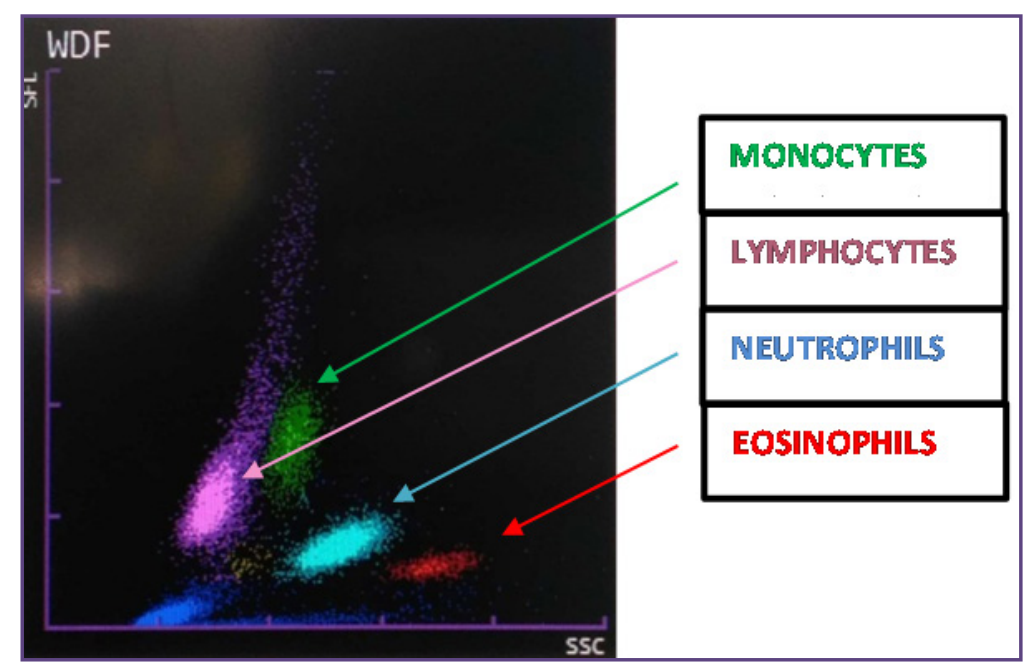

Fig.1: The Normal scattergram.

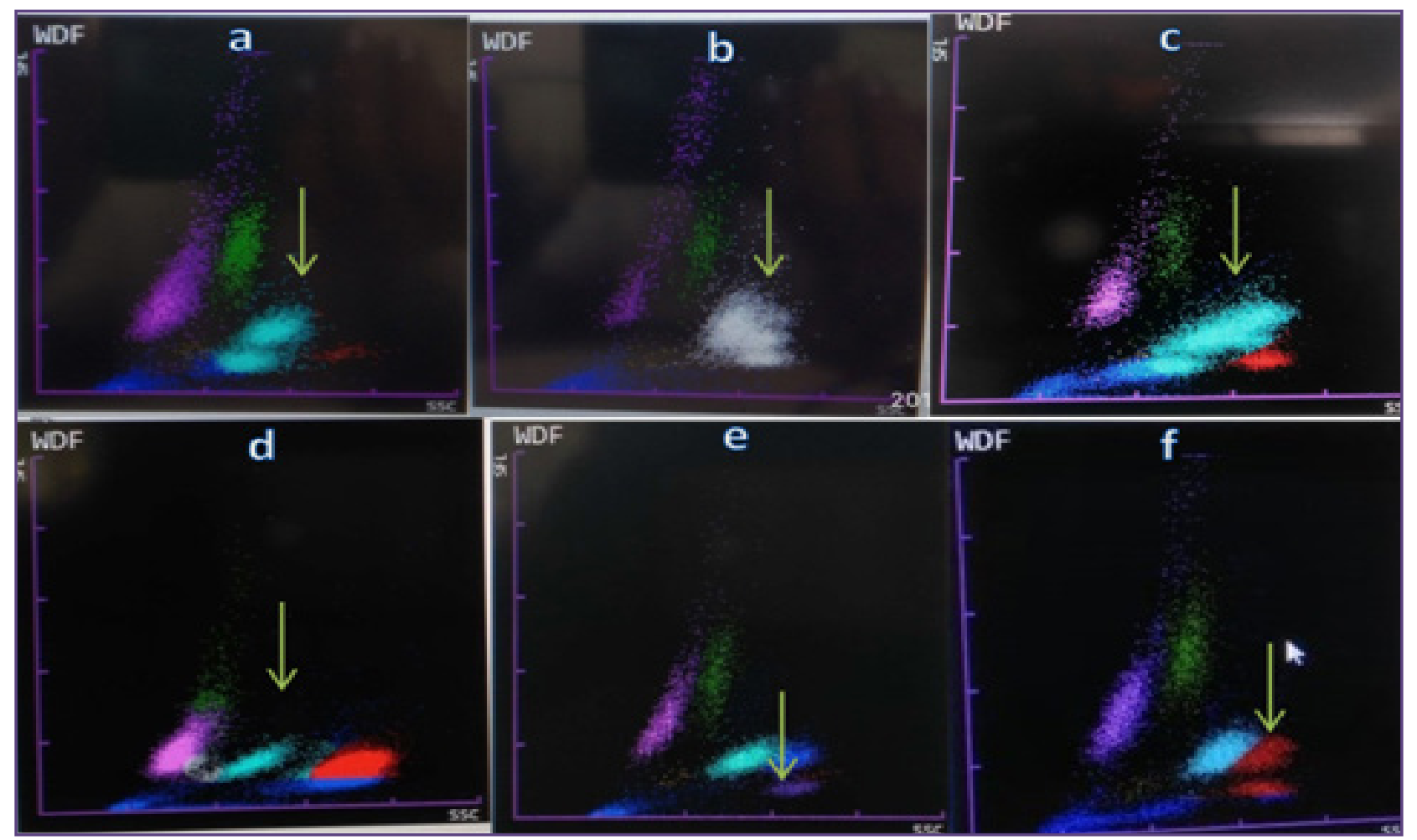

Fig. 2: Patterns of abnormal scattergrams in malarial infection by Sysmex XN-550, (a) Double Neutrophil,(b) Gray Zone,(c) Double neutrophil \& reduced spacing in N \&Eo, (d) Gray zone+DoubleNeutro+reduced spacing in N \&Eo,(e) Abnormal event above $\mathrm{X}$ axis Purple Area, (f)Double Eosinophil- in Malaria infection. 


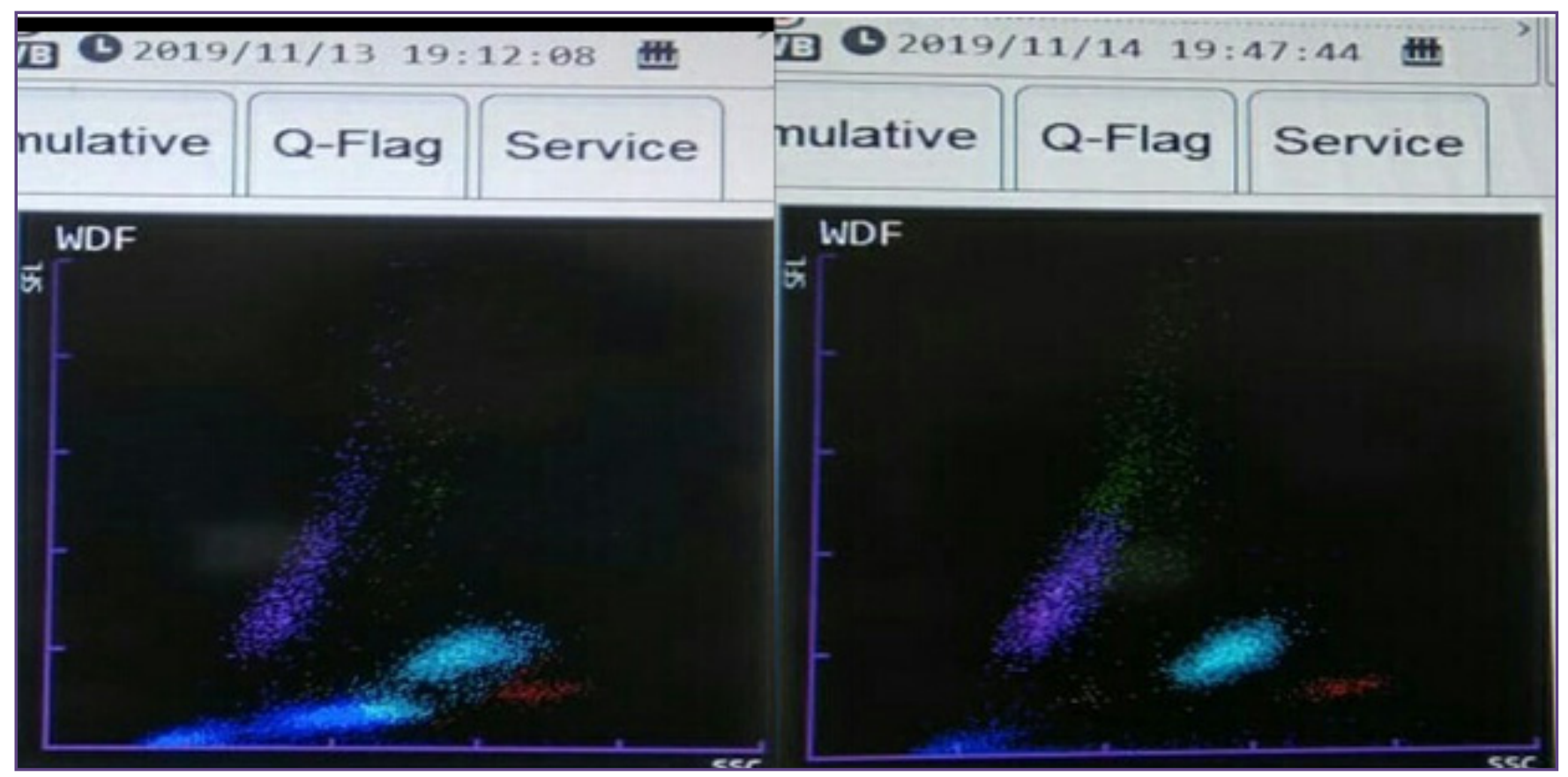

Fig. 3:Pretreatment(Double neutrophil \& reduced spacing in Neutrophil \&Eosinophil) \&post treatmentscattergrams in malaria.

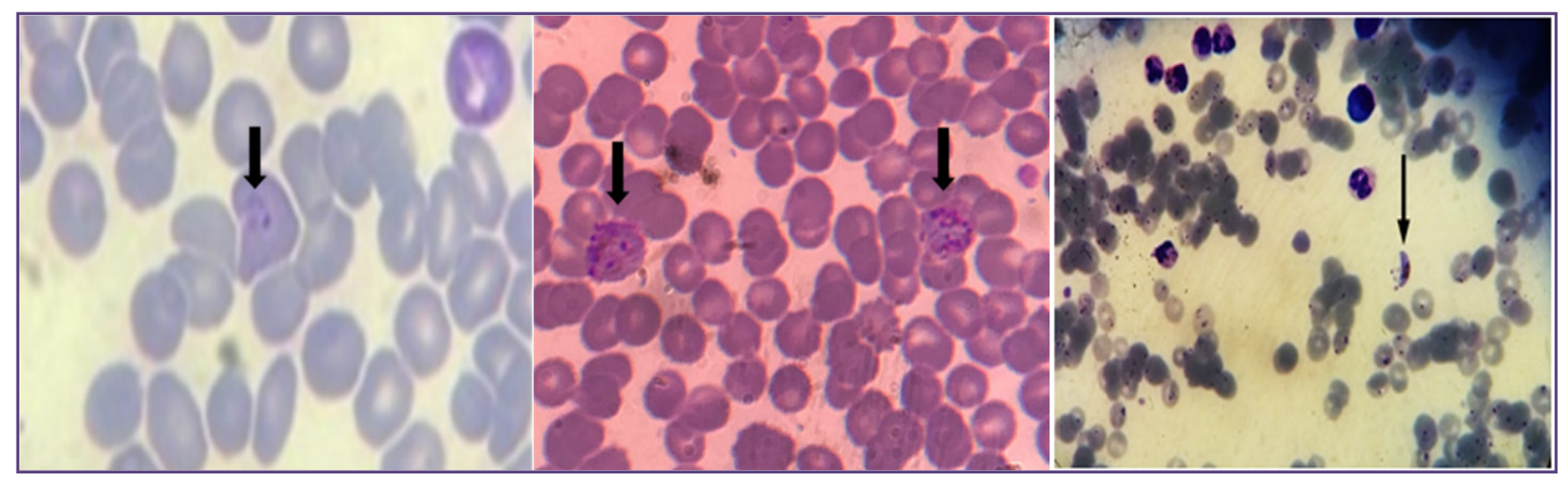

Fig: (4a) Trophozoite of P.vivax, (4b)Schizonts of P.vivax, Fig:(4c) Gametocyte of P.falciparum.

\section{Conclusion}

We observed that scattergram abnormalities are useful in the presumptive diagnosis of malaria. It is impractical to screen manually all peripheral blood films for the diagnosis, especially in our institute where sample load is too high and when the parasitic index is low. Scattergram helps the pathologists and technicians who handle these auto analysers to pick up all suspicious cases and subsequently confirm the same on a PS and other rapid diagnostic tests.

In present study, it was revealed that scattergram of Sysmex XN 550 automated haematology analyser has good sensitivity $(80.19 \%)$, which can be increased to a better level if combined with thrombocytopenia and symptomatology of the patients.

\section{Acknowledgements}

Nil

\section{Funding}

None

\section{Competing Interests}

None declared

\section{References}

1. Tangpukdee N, Duangdee C, Wilairatana P, Krudsood S. Malaria diagnosis: A brief review. Korean J Parasitol. 2009;47(2):93-102.

2. Huh HJ, Jung J, Yoon H, Chae SL. Malaria detection with the Sysmex XE-2100 hematology analyzer using 
pseudoeusinophilia and abnormal WBC scattergram. Ann Haematol2008;87:755- 9.

3. Maru AM, Shrivastava A. Chokshi T, Agnihotri AS. Utility of automated hematology analyzer in diagnosis of malarial parasite.Indian J PatholOncol2019;6(3):428-33.

4. Nguyen PH, Day N, Pram TD, Ferguson DJ, White NJ. Intraleucocytic malaria pigment and prognosis in severe malaria. Trans R Soc Trop Med Hyg 1995;89:200-4.

5. Campuzano ZG, Blair S. Malaria: considerations about diagnosis. Medicina\&Laboratorio. 2010;16:311-354.

6. Yoo JH, Song J, Lee KA, et al. Automated detection of malaria-associated pseudoeosinophilia and abnormal wbcscattergram by the sysmexxe- 2100 hematology analyzer: a clinical study with 1,801 patients and real-time quantitative pcr analysis in vivax malaria-endemic area. The American journal of tropical medicine and hygiene. 2010;82:412-414.

7. Jain M, Gupta S, Jain J, et al. Usefulness of automated cell counter in detection of malaria in a cancer set up-Our experience. Indian journal of pathology \& microbiology. 2010;55:467-473

8. Sharma S, Sethi N, Mukta P, Shivani K, Shivali S. Abnormal WBC scattergram: A clue to the diagnosis of malaria. Hematology. 2013;18(2):101-05.

9. Mohapatra S, Samantaray JC, ArulselviS, Panda J, Munot K, Saxena R. Automated detection of malariawith haematology analyzer sysmex xe-2100. Indian J Med Sci2011;65:26-31.

10. RamyaB,Prashanti G. Role of WBC Scattergram, Histogram and Platelet Indices in Diagnosis of Malaria.National Journal Of Laboratory Medicine.2019;8(4):25-27.

11. Pai Vidya, Shariff M.H. and AijazMuzamil Dar.Malaria diagnosis by abnormal scattergrams in automated hematology analyzer.Int J Pharmacy Biological Sci2016;6(2):55-9.

12. Maina RN, Walsh D, Gaddy C, et al. Impact of infection on haematological parameters in children living in Western Kenya. Malar J. 2010;9(3):4.

13. Gupta P, Guddattu V and KavithaSaravu K. Characterization of platelet count and platelet indices and their potential role to predict severity in malaria.Pathogens and Global Health. 2019;113(2):86-93.

14. Mubeen KH, Devadoss CW, Rangan RA, Gitanjali M, Prasanna S, Sunitha V. Automated hematology analyzers in diagnosis of plasmodium vivax malaria: an adjunct to conventional microscopy. Mediterr J Hematol Infect Dis. 2014;6(1):e2014034.

15. Abro AH, Saleh AA, Abdou AS, Ustadi AM, Shuri HM, Seliem RM. Thrombocytopenia in adults with acute malaria [Internet]. [cited 2019 Aug 17]. Available from:https://pdfs.semanticscholar.org/d85a/ da9ca0d403cc9b7b8444c85a886ff91495d6.pdf

16. Chandra S, Chandra H. Role of haematological parameters as an indicator ofacute malarial infection in Uttarakhand state of India.Mediterr J Hematol InfectDis. 2013;5(1).

17. Clark IA, Chaudhri G. Tumour necrosis factor may contribute to the anemia of malaria by causing dyserythropoiesis and erythrophagocytosis. Brit J Haematol. 1988;70:99-103.

18. Angus BJ, Chotivanich K, Silamut K, Ruangveerayuth R, Hardeman MR. Red blood cell deformability as a predictor of anemia in severe Falciparum malaria. Am J Trop Med Hyg. 1999;60:733-37.

19. SinghA, Narang V, Sood N, Garg B, Gupta VK. Malaria diagnosis usingautomated analysers: A boon for hematopathologists in endemic areas. J ClinDiagnostic Res [Internet]. 2015 [cited 2019 Oct 4];9(10):EC05-8. Availablefrom:https://www.ncbi. nlm.nih.gov/pmc/articles/PMC4625244/

20. Adlekha S, Jaiswal R, Chadha T, Singla A.The correlation of spuriouseosinophilia in automated hematological analyzer sysmex Xs-800i with plasmodium infection diagnosis.Indian J Med Sci [Internet]. 2011 [cited 2019 Oct 4];65(11):469-75.

*Corresponding author:

Dr. Rajeshwar Suresh Bute, L-103, Mhada Colony, Behind Durga Lawn, Kaulkhed, Akola. 444004

Phone: +91 9021170789

Email: rajbute@gmail.com

Date of Submission : 26/11/2020

Date of Revision $\quad: 25 / 12 / 2020$

Financial or other Competing Interests: None.

Date of Acceptance : 04/01/2021

Date of Publication : 30/01/2021 